Revue d'histoire de l'Amérique française

सES REVUE D.HISTOIRE DE L'AMÉRIQUE FRANÇAISE

\title{
L'histoire de la médecine : une porte ouverte sur l'histoire sociale
}

\section{André Paradis}

Volume 42, numéro 1, été 1988

URI : https://id.erudit.org/iderudit/304651ar

DOI : https://doi.org/10.7202/304651ar

Aller au sommaire du numéro

Éditeur(s)

Institut d'histoire de l'Amérique française

ISSN

0035-2357 (imprimé)

1492-1383 (numérique)

Découvrir la revue

Citer cette note

Paradis, A. (1988). L'histoire de la médecine : une porte ouverte sur l'histoire sociale. Revue d'histoire de l'Amérique française, 42(1), 73-83.

https://doi.org/10.7202/304651ar d'utilisation que vous pouvez consulter en ligne.

https://apropos.erudit.org/fr/usagers/politique-dutilisation/ 
NOTE CRITIQUE

\title{
L'HISTOIRE DE LA MÉDECINE: UNE PORTE OUVERTE SUR L'HISTOIRE SOCIALE
}

\author{
ANDRÉ PARADIS \\ Département de philosophie \\ Centre de recherche en études québécoises \\ Université du Québec à Trois-Rivières
}

Il y a sans doute bien des façons de «faire l'histoire». Généalogiquement, il y a d'abord l'histoire édifiante, ou héroïque, qui consiste à reproduire au coeur même de l'historiographie les valeurs fondatrices d'une communauté humaine et à réaffirmer le rapport à la tradition, à l'origine. C'est l'histoire de la fidélité à la mission. Cette façon de faire l'histoire privilégie la continuité à l'encontre des perspectives d'instabilité et de contingence du Nouveau-Monde. Comme dans les chroniques monastiques médiévales, l'événement ne vaut ici que parce qu'il incarne sur un mode répétitif un ordre symbolique qui le transcende. Le temps de l'histoire s'enracine dans l'éternité. L'histoire des communautés hospitalières, des Hôtel-Dieu et des Hôpitaux Généraux, celle d'un Mgr de Saint-Vallier par exemple, seraient partie prenante de cette façon de faire où semble primer la mémoire. L'histoire serait d'abord et avant tout le déroulement et l'accomplissement d'un projet. Écrire l'histoire, serait témoigner de cet accomplissement. Il y a en second lieu l'histoire empirique, dont la prétention est de délester l'événement de sa charge mythique et de le restituer dans sa crudité factuelle. «History is a matter of fact.» Les historiens classiques de la médecine québécoise, les Ahern, Abbott, Heagerty, McDermot, Leblond, Boissonnault et Lewis par exemple, feraient partie de ces historiens du factuel et de l'archive où la romance et le rituel fondateur semblent tombés en désuétude. L'histoire de la médecine, c'est ici l'histoire des faits et gestes de la profession et la succession chronologiquement orchestrée des événements marquants de l'institutionnalisation des pratiques médicales. Le fondement de l'histoire, c'est l'authenticité du document qui atteste le fait. La troisième façon de faire l'histoire consiste à passer du 
fait à l'interprétation. L'histoire, c'est d'abord et avant tout une façon de lire le document. On ne saurait s'en cacher. Et l'historien ne peut prétendre s'exclure de sa lecture. Il n'y a donc plus ici de vérité intrinsèque du factuel. Le fait ne vaut que parce qu'il signifie ou donne à signifier pour l'historien. Et il ne donne à signifier que pour autant qu'il appartient lui-même à une écologie: écologie socio-culturelle, écologie socio-politique, écologie épistémique, écologie des pratiques scientifiques et des interactions du savoir propre à un espace et à un temps donnés de l'histoire. De même qu'il n'y a pas de texte sans contexte, il n'y aurait pas d'événement sans un réseau complexe de conditions sociales ou discursives d'émergence. L'historien des sciences et de la médecine serait donc lui aussi, à long terme, un architecte des grands ensembles, des mentalités, des positivités discursives sur lesquels viendrait s'articuler et prendre forme le sens même de l'événement singulier. Nous serions entrés dans l'ère des discontinuités et des structures profondes où le factuel, le fait à l'état brut, n'apparaît désormais que comme phénomène de surface. Loin de porter en lui-même sa signification ou sa justification, la tâche de l'historien consisterait plutôt à en rendre compte, à révéler la logique qui le sous-tend. C'est-à-dire à l'interpréter par autre chose que lui-même. La question centrale de l'historien ne serait pas: «qu'est-ce qui s'est produit» mais plutôt «pourquoi et comment cela a-t-il pu se produire». Dans l'ensemble, l'histoire serait donc, elle aussi, pour emprunter à Edgar Morin, une affaire de complexité croissante.

C'est peut-être dans ce troisième âge interprétatif de l'histoire que cherche à nous faire entrer l'ouvrage de Fournier, Gingras et Keel ${ }^{1}$, l'histoire des sciences et de la médecine québécoises étant demeurée en marge de l'évolution générale de la discipline historique depuis de nombreuses années. Ce n'est pas que l'ouvrage Sciences et médecine au Québec soit particulièrement explicite sur son objet, même s'il se trouve sous-titré, de façon un peu lâche, perspectives sociohistoriques. Hormis l'introduction un peu rapide du texte de Fournier, on n'y trouve pas en effet d'éléments de réflexion spécifiant en quoi l'institution médicale, les connaissances scientifiques ou la maladie comme réalité sociale peuvent être un objet pour l'histoire. Pas plus qu'on ne voit clairement articulé, en termes d'éclairage, le rapport entre la médecine, les sciences et le pouvoir socio-politique. Sous ce rapport, l'ouvrage pourrait donner à penser que nous avons surtout affaire ici à un collage de textes, les auteurs ayant eux-mêmes voulu «respecter la diversité des préoccupations et des perspectives de chacun des participants». C'est un fait que nous sommes encore bien loin ici d'un volume intégré sur l'histoire

1 Marcel Fournier, Yves Gingras et Othmar Keel, Sciences \& médecine au Québec, perspectives sociohistoriques. Québec, Institut québécois de recherche sur la culture, 1987. $210 \mathrm{p}$. $20,00 \$$. 
de la médecine au Québec. Pourtant, à bien lire cet ouvrage, sobrement écrit, méticuleux, richement étoffé et documenté, à bien le parcourir, on se rend vite compte qu'il y a malgré tout une double visée de congruence qui l'anime. D'une part, il s'agit d'illustrer que la science est partie prenante de l'histoire sociale. Les connaissances médicales, en effet, ont elles aussi leur cadres sociaux et c'est en eux qu'elles trouvent leurs conditions structurelles aussi bien que dynamiques de possibilité. Une fois cela admis, on est en droit de penser que le développement des sciences et de la médecine ne peut être jaugé à la seule mesure des grandes déterminations de l'économie libérale; il faut procéder ici à une démarche plus fine, plus sophistiquée, qui prend en considération la dynamique même des institutions culturelles; car c'est bien dans ces institutions que se forme la perception du monde et, du même coup, le regard que l'on porte sur les choses, sur les phénomènes, fussent-ils physiques, biologiques, psychiques ou sociaux. La formation du regard scientifique, en l'occurrence médical, tient ici aux idéaux et aux buts culturellement assignés à l'activité de connaissance, aux protocoles d'apprentissage et de transmission des savoirs, à la structuration et à la légitimation de l'expérimentation et de la recherche. Mais elle n'est pas indifférente pour autant aux importations et aux contacts culturels pas plus qu'elle n'échappe à la dominance ou à la résistance des savoirs et des pouvoirs en place. Sorte de sociologie historique de la connaissance scientifique si l'on veut, plus conjoncturelle et moins typologique que celle d'un Gurvitch, et qui nous conduirait à un second constat: car c'est bien alors à deux histoires communautaires des sciences et de la médecine que nous aurions affaire au Québec: l'une anglophone, davantage favorisée, au 19e siècle, par les acquis encore tout chauds de la philosophie expérimentale et positiviste, toute gagnée à l'esprit d'invention et à la quête d'applications technologiques; l'autre francophone, plus spéculative et ornementale, éprise de théorie et de vulgarisation pour autant que ces dernières sont destinées à meubler l'esprit et à «parfaire» la formation classique du parfait honnête homme. Dans les faits cependant, une tension soutenue, conflictuelle, entre le libéralisme et le «classicisme», entre l'innovation et la tradition, qui fluctue au fil même de nos rapports sociétaux; la société canadienne-française cherchant finalement à surmonter le handicap de sa situation de minorité et à rattraper le «temps perdu». On comprend mieux alors qu'après une longue période de tergiversations et d'hésitations conservatrices en faveur de la vraie science, celle qui ne dérange pas trop, qui n'est ni darwiniste, ni saxonne, ni franc-maçonne et qui sait composer avec la foi, on ait pu se mettre à parler plus volubilement encore, dès le lendemain de la première grande guerre, du retard du Québec francophone en matière d'économie et de connaissances scientifiques. Un retard, nous dit-on, que des intellectuels comme Jacques Rousseau, Georges Baril, Adrien Pouliot et Cyrias Ouellet pressentaient avec inquiétude autour des années 1930 et qu'il ne sert plus à rien de décrier. Il vaudrait 
mieux chercher maintenant à comprendre. Une sociologie différentielle de nos institutions culturelles et scientifiques, attentive aux rythmes et aux interactions propres à chaque milieu, francophone et anglophone, aiderait en ce qu'elle ferait peut-être de l'idée de retard culturel un produit douteux de l'esprit. Finalement, ce que l'ouvrage dirigé par Fournier, Gingras et Keel nous propose, ce n'est pas tant une histoire de la médecine québécoise, celle-ci restant encore à faire, mais des balises et quelques analyses de cas significatifs où l'on semble enfin «prendre possession» du terrain. Par le fait même, c'est peut-être aussi un modèle d'analyse que nous livre cet ouvrage.

La science et la médecine étant parties prenantes de l'histoire sociale, c'est donc à une relecture de l'histoire du Québec, du point de vue des conditions socio-historiques de la production du savoir que les auteurs nous convient. L'introduction pose la question de notre intérêt mitigé pour les sciences et pour l'histoire des sciences. Elle fait l'historique des inquiétudes et des interrogations qui ont jalonné le premier tiers du 20e siècle en milieu francophone. Après quoi les textes de Robert Gagnon et de Richard Jarrell nous introduisent au coeur même de l'histoire. L'apport de ces deux premiers textes, quelque peu redondants en ce qui concerne l'historique de l'idéologie ultramontaine, tient surtout au fait qu'on y élargit notre compréhension de cette «vision du monde». Les considérations sur la science en feraient étroitement partie. L'idéologie ultramontaine, dont les racines antilibérales nous sont rendues explicites par Jarrell, ne pouvait qu'entraîner avec elle un partipris contre les progrès du «matérialisme» bourgeois et les prétentions du rationalisme scientifique. Le lien semble en tout cas étroit entre l'opposition cléricale au libéralisme et l'opposition à la science et au progrès technique même si, apparemment, le clergé a toujours fait preuve d'une très grande circonspection en ces matières. Si la conclusion du texte de Jarrell nous propose une interprétation «nuancée» de la position des intellectuels cléricaux à l'endroit de l'enseignement des sciences au Québec, il faut regretter qu'elle ne nous éclaire pas vraiment sur le sens de l'ambivalence du clergé qui, sans être contre la science, ne semble pas pour autant être pour. On pourrait interpréter cette ambivalence comme un repli stratégique, comme une acceptation à contre-coeur, comme un refus déguisé ou comme une marque d'ouverture réelle, bien que modérée, vis-à-vis les nouvelles valeurs de la connaissance «naturelle». Tout dépend manifestement de ce que l'on comprend soi-même sous l'appellation de «science». Thomas d'Aquin n'hésitait pas à parler de la théologie comme science. Il n'y a pas d'obstacle à ce que le clergé du 19e siècle, néo-thomiste de formation, ait plutôt pensé en termes de vraie science. Nul doute pourtant que l'adoption d'une position même modérément favorable à l'endroit des sciences et de la technologie nouvelle ne pouvait que porter atteinte, directement ou indirectement, aux qualifications et aux valeurs du clergé et remettre en question son statut 
au sein des institutions culturelles canadiennes-françaises. Même si les enjeux ne pouvaient alors être clairs, le haut clergé catholique, bien informé, pouvait-il l'ignorer? Et dans quelle mesure était-il disposé à emprunter les voies de la «modernité»?

À cet effet, Gagnon ramène en surface, pour notre bénéfice, des textes inconnus ou mal connus du $19 \mathrm{e}$ siècle. Ces textes nous informent sur les divergences qui opposaient alors le clergé et certaines fractions de l'élite journalistique ou politique en matière d'éducation. Ces textes nous parlent de l'engorgement des professions libérales, de la pénurie d'ingénieurs et d'industriels en milieu canadien-français, de l'incurie de l'Église en regard de l'éducation pratique et des sciences appliquées, de l'anachronisme du grec et du latin. Des critiques caustiques, exprimées parfois vertement, et qu'on croyait propres au 20e siècle. Des critiques qu'on ne faisait encore que répéter, sans trop s'en rendre compte, à l'époque du rapport Parent. On en conclut que l'idée d'un consensus «quasi-unanime» au sein de l'élite laïque francophone du $19 \mathrm{e}$ siècle, soi-disant inféodée à l'Église, ne fut peut-être qu'une interprétation abusive: même après les troubles de 1837.

Le texte de Yves Gingras vient illustrer de façon fort ingénieuse les implications des deux premiers textes. L'auteur y montre comment la perception de l'enseignement et de la recherche scientifiques en milieux francophone et anglophone induisit, en 1896, des réactions fort différentes dans l'accueil que l'on fit, au Québec, à la découverte de Roentgen. D'un côté, les rayons X amenèrent les physiciens de McGill à perfectionner immédiatement la technique de l'exposition, à pousser la recherche du côté des propriétés physiques et germicides des rayons, à collaborer avec les médecins en vue de leur utilisation pratique en chirurgie et à publier les résultats de ces initiatives dans les revues savantes. En milieu francophone au contraire, les physiciens «religieux» de l'Université Laval, qui ne recouraient pas encore au laboratoire dans le cadre de leur enseignement, se livrèrent plutôt à la simple «reproduction» de l'expérience et à sa diffusion «mondaine». L'intérêt du texte de Gingras tient surtout au fait qu'il montre en quoi la formation des chercheurs et l'infrastructure même de la recherche puisaient déjà ici à des sources et à des traditions nettement différentes: l'une locale, fortement enracinée dans le réseau des collèges classiques et ne débouchant pas encore sur des emplois spécialisés, l'autre britannique et déjà tributaire des habitus de recherche développés à Cambridge par Maxwell et Thomson et dont Callendar et Rutherford allaient être les relayeurs locaux à McGill. S'il faut regretter ici quelque chose, c'est que Gingras n'ait pas appuyé son hypothèse des «deux solitudes scientifiques» sur une étude comparative plus poussée des tout débuts de la radiologie dans les milieux scientifiques et hospitaliers anglophone et francophone. Le dépouillement du Bulletin médical de Québec et de $L a$ revue médicale fournirait sans aucun doute des précisions supplémen- 
taires sur l'intérêt des médecins canadiens-français pour la radiologie naissante. Boissonnault, pour sa part, fait état d'un recours assez précoce à la radiologie à l'Hôtel-Dieu de Québec au tout début du 20e siècle. En d'autres termes, il n'est pas dit que l'intérêt pour la radiologie en milieu hospitalier canadien-français ait suivi un développement parallèle à celui des enseignants en milieu académique. Il faudrait voir de plus près. Je porte à l'attention de Gingras qu'en ce qui concerne le milieu anglophone, David Sclater Lewis a lui aussi consacré quelques pages intéressantes aux tout débuts de la radiologie montréalaise dans sa monographie sur l'Hôpital Royal Victoria ${ }^{2}$.

Farley, Keating et Keel nous entretiennent quant à eux des politiques de prophylaxie antivarioliques à Montréal dans la seconde moitié du $19 \mathrm{e}$ siècle. La prévention des épidémies de variole par la vaccination obligatoire nous renvoie à une histoire fort complexe où les enjeux proprement scientifiques et médicaux se trouvent étroitement associés à des intérêts de toutes sortes, aussi bien économiques que professionnels, ethniques et politiques. L'histoire des politiques de vaccination est par ailleurs incompréhensible si on ne la replace pas dans le cadre beaucoup plus général du processus d'urbanisation et d'implantation de l'économie de marché, les épidémies, en milieu densément peuplé, ayant une incidence non négligeable sur le cours de la production et sur les finances publiques. Il est indéniable en tout cas que nous avons affaire ici à un cas d'espèce où l'étude des articulations entre la science et la société, entre la connaissance et l'idéologie, trouve l'une de ses plus parfaites concrétisations. Même s'il y a encore ici matière à interprétation, on doit à Farley, Keating et Keel d'avoir su débrouiller les innombrables péripéties, parfois houleuses, qui ponctuent la mise en oeuvre de ces politiques de prophylaxie. On leur doit aussi d'avoir fait valoir la multiplicité des facteurs qui doivent être pris en considération dans l'appréciation des positions médicales ou populaires pour ou contre la vaccination. Les résistances farouches à la politique de vaccination n'étaient pas en tout cas l'affaire exclusive des Canadiens français. Le mouvement des antivaccinateurs était international, les médecins anglophones, tout comme les francophones, ne faisaient pas l'unanimité entre eux; la coercition civile faisait elle-même accroc aux libertés individuelles chères à l'idéologie libérale et surtout aux habitudes de «solidarité» familiale chères aux Canadiens français. On peut penser de même que la piètre qualité des vaccins, l'incertitude quant à leur véritable nature, le manque de précautions antiseptiques et les accidents inoculatoires n'étaient pas tout à fait étrangers à la méfiance collective. Le texte de Farley, Keating et Keel vient donc ici désamorcer la tentation d'extrapoler trop rapidement et de réduire le débat à une simple

2 David Sclater Lewis, Royal Victoria Hospital, 1887-1947 (Montréal, McGill University Press, 1969), 155-159 et 210-214. 
question de psychologie ou d'ignorance ethnique et collective; ou encore à une confrontation carrément politique entre l'assurance des colonisateurs, favorables à la découverte de Jenner, et le ressentiment des colonisés, hostiles à la science anglaise. Il faut dire cependant que derrière la multiplicité des facteurs fort justement allégués par Farley, Keating et Keel, on n'arrive pas à cerner ce qui pourrait expliquer la vitalité conjoncturelle de l'opposition aux politiques de vaccination et en particulier la vitalité du mouvement des antivaccinateurs. Qu'est-ce qui pouvait pousser, par exemple, le docteur Coderre (et ses associés Dagenais, Crevier et al) à militer en zélateur contre la politique de vaccination obligatoire? Question de formation médicale traditionnelle qui le rendait inapte à «saisir» la logique assez paradoxale, avouons-le, du «poison-vaccin» qui immunise (l'idée d'immunisation par le pus vaccinal étant incompatible avec les théories pré-pastoriennes)? Question de personnalité conservatrice et profondément réactionnaire en regard des progrès du savoir (Coderre, qui écrit très peu, se découvre soudainement une vocation de polémiste et d'écrivain à propos de la vaccination)? Question de tiraillements professionnels ou d'hostilité personnelle contre la bonne fortune des vaccinateurs publics qui s'alimentent à même les deniers publics? Question de lutte d'influence entre l'élite médicale traditionnelle canadienne-française (dont Coderre est un des principaux représentants) et la fraction médicale progressiste dirigée par un personnage aussi proéminent médicalement et politiquement que Hingston? Question de schisme et de clivage ethno-linguistique de plus en plus marqué à partir des années 1875 entre les membres francophones et anglophones de la profession (qui se départagent entre la Société médicale de Montréal et la Montreal Medico-Chirurgical Society)? Il me semble que nous faisons face ici à un problème (puzzle) fort complexe et intéressant qui impliquerait une réévaluation de la conjoncture et des tendances (ethno-linguistiques, intellectuelles, associatives) qui unissent et divisent la profession médicale au Québec.

À ceux qui s'interrogent sur les facultés de médecine, sur leurs politiques de recrutement et leur insertion en terre québécoise depuis plus de 150 ans, l'étude comparative menée par George Weisz apporte, sinon des réponses évidentes, du moins des indications fécondes et suggestives. L'étude de Weisz tend à démontrer que chaque école de médecine au Québec répondit à une vocation socio-économique et sociopolitique spécifique et à un bassin de clientèle relativement stable et défini. Seule la faculté de médecine de l'Université McGill, la plus ancienne et la plus pressée aussi par la concurrence de ses nouvelles rivales des années 1870 (l'École de médecine et de chirurgie et la faculté de Bishop's College de Lennoxville) eut à composer avec un bassin mobile. Puisant majoritairement sa clientèle à Montréal et en Ontario jusque dans les années 1890, concurrencée à nouveau par l'Université de Toronto à compter de 1887, récupérant avec les Maritimes et avec 
l'Ouest canadien, McGill se tournera ensuite vers les candidats américains dont le nombre, à compter de 1925, débordera largement la capacité d'accueil des écoles médicales de nos voisins du sud. Le nombre de ces écoles déclinant rapidement par surcroît, on comprend alors que les étudiants américains virent dans la faculté de McGill une alternative valable en regard des écoles médicales de prestige, telles celles de Philadelphie, de Boston et de New York. On saisit mieux encore pourquoi la faculté de médecine de l'Université McGill, déjà réputée et bien soutenue financièrement par les dons privés, put alors contingenter sa clientèle, miser sur l'excellence et relever considérablement ses normes d'admission. On alla même, dit-on, jusqu'à exiger des nouveaux candidats l'équivalent d'un diplôme universitaire de premier cycle en sciences. Le clivage entre la formation scientifique des étudiants anglophones et celle des francophones trouverait sans doute là l'une de ses nombreuses explications. Même si le statut et la vocation socio-politique des différentes écoles médicales québécoises demeure à préciser en fonction d'une mise en contexte socio-politique plus large, l'étude statistique de Weisz, en signalant des constantes et des écarts significatifs, ne va pas sans suggérer, au-delà de la lettre, des pistes de réflexion et des prolongements intéressants. Il serait en l'occurrence intéressant d'interroger l'impact qu'a pu exercer sur l'évolution des idées et de l'enseignement de la médecine anglophone québécoise l'ouverture de McGill sur les États-Unis, bien avant 1925 (on sait par exemple que, déjà au $19 \mathrm{e}$ siècle, Osler, l'un des plus éminents praticiens québécois, avait pratiqué à John Hopkins). Le clivage ethno-linguistique entre la médecine anglophone en rapport d'osmose active avec les idées et le personnel médical étrangers anglophones (via les étudiants diplômés) et la médecine francophone confinée à des contacts culturels beaucoup plus limités pourrait peut-être expliquer le dénivellement qualitatif que l'on observe, au niveau de la médecine clinique en particulier, entre les périodiques médicaux francophones et anglophones du 19e siècle, surtout à compter de 1880 .

En nous proposant une étude sur l'Institut du Cancer de Montréal, Marcel Fournier attire enfin notre attention sur une institution paramédicale française et québécoise. Il entend du même coup illustrer par ce biais l'univers fort complexe de la régulation et de l'action sociale qui, nous dit-il, «donne à l'activité scientifique sa configuration particulière». Ainsi le contenu et la nature même des recherches biochimiques, biophysiques et épidémiologiques sur le cancer se trouvent-ils à la fois réglés et sanctionnés par les retombées sociales anticipées, les buts et les stratégies assignées à la recherche, les modalités de financement, les politiques d'évaluation qualitative des «actes» de recherche, le recrutement du personnel et les interactions socio-culturelles qui déterminent le profil de chaque période de croissance institutionnelle. Les remaniements opérés dans l'orientation de la recherche ne sont 
jamais étrangers aux difficultés et aux succès rencontrés dans l'un ou l'autre de ces domaines. La position, le statut et l'efficacité des chercheurs s'en trouvent d'autant favorisés ou inhibés. Ou encore est-ce une découverte scientifique obtenue dans un contexte institutionnel étranger qui vient remettre en question le dispositif de recherche déjà mis en place. C'est donc l'évolution d'un Institut du Cancer bien concret, tendu entre les préoccupations cliniques et la recherche fondamentale, entre l'hôpital et l'université, entre la science enseignée et la science appliquée, entre la chimiothérapie conventionnelle et la transcriptase, entre le ministère des Affaires sociales et l'Institut national du Cancer que tente de retracer pour nous Marcel Fournier. Il le fait en tenant compte des personnes, des réalisations et des conjonctures qui ont modelé l'Institut, sans omettre quelques «bruits» qui ont fait vibrer la machine. Ici encore, on saisit un peu mieux en quoi l'histoire des sciences appliquées est étroitement solidaire de l'histoire sociale. En ce sens on peut dire qu'il n'y a jamais d'autarcie ou d'auto-suffisance sectorielle de la science. Le médecin, le chimiste ou le biologiste n'existent pas par la seule grâce d'un lieu d'étude encyclopédique qui leur pré-existerait et dont ils ne seraient que les dépositaires attitrés. Ils n'existent bien que parce qu'on ne cesse de leur apporter des problèmes qui sont bel et bien des problèmes de société et de leur demander d'urgence des solutions déjà élaborées ou inédites. L'histoire de la médecine fournit de nombreux cas de parallélisme entre la recherche médicale et les problèmes de santé communautaires. Les publications médicales du $19 \mathrm{e}$ siècle québécois sur les maladies infectieuses à caractère épidémique par exemple (du choléra à la syphilis en passant par la tuberculose) illustrent particulièrement bien la coïncidence entre les intérêts de la recherche médicale et les attentes pressantes de la société. De la même façon, on pourrait dire que le développement de l'obstétrique et de la gynécologie avec un Lapthorn-Smith et de l'hygiène avec un Desroches devient significatif si on le met en rapport avec les taux particulièrement élevés de mortalité infantile et avec les conditions de vie déplorables qui prévalent dans le milieu urbain montréalais du $19 \mathrm{e}$ siècle. Les solutions recherchées à de tels problèmes impliquent des stratégies de développement et d'information proportionnelles aux ressources et aux limites propres à chaque communauté socio-politique. C'est ici, à vrai dire, que la science rejoint l'intelligence ou la caducité de l'administration politique.

Bernier notait récemment ${ }^{3}$, avec beaucoup de réserves, une recrudescence d'intérêt pour l'histoire de la médecine en milieu francophone depuis une dizaine d'années. Il peut y avoir à cela au moins deux raisons. C'est peut-être d'une part qu'on peut maintenant asseoir l'histoire

3 Jacques Bernier, «Scientia Canadensis», Revue d' histoire des sciences, des techniques et de la médecine au Canada, 33. 
de la culture scientifique sur une meilleure connaissance de l'histoire sociale et des idéologies. Et c'est peut-être aussi qu'on se rend compte de plus en plus que l'histoire de la médecine, loin d'être une histoire de luxe, est, elle aussi, une porte d'entrée privilégiée sur l'histoire sociale et culturelle. L'histoire de la médecine n'est pas plus étrangère aux grands courants idéologiques qu'elle n'est étrangère à l'imaginaire socioculturel de façon générale. La pratique médicale hérite en effet de beaucoup de croyances morales, religieuses et politiques et elle-même nourrit souvent la culture globale de son propre imaginaire «scientifique». La connaissance scientifique, objectivante, quant à elle n'est jamais un produit spontané de l'esprit. Elle implique des cheminements laborieux, tortueux, pleins de heurts et de limites à respecter et à franchir de même que des stratégies de persuasion, des regroupements et des alliances pour imposer et faire valoir de nouvelles théories, de nouveaux contenus d'enseignement, de nouvelles façon de gérer les corps malades qui font problème pour eux-mêmes et pour les autres. La maladie interprétée et administrée est une entreprise en même temps que l'objet de préoccupations humanitaires. Et le savoir est aussi pouvoir. Pouvoir de soulager ou de guérir, mais aussi pouvoir de concourir à la législation et à la régulation de l'espace et des rapports sociaux. L'ouvrage de Fournier, Gingras et Keel, par la largeur heuristique des hypothèses «socio-politiques» qui y sont formulées, a sans doute le mérite de concourir à une vision plus compréhensive de l'histoire médicale.

La médecine étant une discipline de convergence, on conçoit fort bien que les revues médicales et les archives des facultés de médecine, des instituts de recherche et des associations médicales pourraient donner lieu à beaucoup d'autres travaux historiques similaires à celui de Fournier, Gingras et Keel. Des travaux susceptibles de nous instruire sur l'histoire de la chimie, de la biologie, des sciences naturelles et de la physique comme sciences appliquées et impliquées jusque dans nos représentations les plus quotidiennes du corps et de son écologie naturelle. Mais aussi des travaux susceptibles de nous informer plus adéquatement sur nos médecins comme producteurs ou consommateurs de savoir, sur leur enracinement ou leur déracinement culturel, sur leurs initiatives, leurs stratégies d'innovation et, pourquoi pas, sur les raisons de leur «inertie» ou de leur «retard» relatif dans le champ de la connaissance et des pratiques scientifiques. Que savons-nous d'un Hingston, d'un Fafard, d'un Desroches, d'un Laberge, d'un Séverin Lachapelle et de leur poids relatif dans le développement de la médecine, de son enseignement et des institutions de santé au Québec? Le sachant un peu mieux, peut-être serions-nous alors à même de mieux saisir toutes ces «subtilités» et ces enjeux qui font de la culture scientifique non seulement un lieu de pouvoir mais aussi, inextricablement et conjointement, un lieu de représentation, de savoir et de «perception du monde». C'est peut-être dans l'histoire des sciences que se jouent, plus qu'on 
n'ose le croire, l'histoire et le devenir de notre société depuis la seconde moitié du 19e siècle. Le capitalisme et le libéralisme, comme le soutiennent Marx, Weber et Merton, ont construit leur empire sur les notions «solidaires» de travail, de progrès et de bien-être. Si la médecine participe de cette dernière notion, elle est aussi inséparable de celle de progrès. Et celle-ci, à son tour, est inséparable des stratégies sociales de la connaissance. 